\title{
HÁBITOS ALIMENTICIOS RELACIONADOS CON CARIES Y GINGIVITIS
}

\section{EN NIÑOS DE 6-12 AÑOS EN INSTITUCIONES EDUCATIVAS DE OROPESA; CUSCO 2014.}

Yahaira Paola Vargas Gonzáles'1, Nelly Valdez Aduviri², Gladys Jara Pacheco ${ }^{3}$.

\section{RESUMEN}

En la última década, las enfermedades de mayor prevalencia en la cavidad bucal según la OMS y la OPS son la caries dental, y la enfermedad periodontal siendo estos, factores de riesgo para las enfermedades cardiovasculares, respiratorias, gastrointestinales que afectan a los sistemas del cuerpo humano. La OMS declaró que del 60 al 90\% de los escolares de todo el mundo padecen de caries dental y el $38.7 \%$ padece de gingivitis. La salud bucal es importante para el desarrollo físico y mental de las personas ya que

1 Doctora. Docente Auxiliar, Escuela Profesional de Odontología de la Universidad Nacional de San Antonio Abad del Cusco. E-mail: yhajaod@hotmail.com

2 Doctora. Docente Auxiliar, Escuela Profesional de Odontología de la Universidad Nacional de San Antonio Abad del Cusco.

3 Cirujano Dentista, Escuela Profesional de Odontología de la Universidad Nacional de San Antonio Abad del Cusco. 
desempeña un papel importante en la calidad de la vida. Sin embargo, la caries dental y la enfermedad periodontal aparecen entre las primeras causas de morbilidad bucal a nivel mundial y nuestro país no está libre de estos problemas. Métodos: Fue un estudio descriptivo, prospectivo y transversal, cuya muestra estuvo constituida por 248 niños de las I.E del Distrito de Oropesa. Resultados: Se encontró una alta prevalencia la caries grave en un $64.3 \%$ seguido de la caries moderada en un $29.5 \%$; debido al elevado consumo de alimentos ricos en azucares extrínsecos. La frecuencia del consumo de alimentos cariogénicos fue alta $(63 \%)$ y la de alimentos protectores fue baja en un 55\%. El sexo masculino presento caries dental en un $34.4 \%$ y el sexo femenino presento mayor porcentaje de gingivitis en un $25.4 \%$. Conclusiones: Los hábitos alimenticios están relacionados con la prevalencia de caries, debido al elevado consumo de alimentos ricos en azucares extrínsecos, también está relacionado con la gingivitis; esto se debe a la falta de conocimientos sobre la higiene oral, así mismo se encontró una mayor prevalencia de la caries grave y de una gingivitis leve.

PALABRAS CLAVE: Hábitos alimentarios, Caries dental y Gingivitis.

\section{"Diversos factores predisponen al individuo a adquirir caries, como: la calidad de la dieta, la misma que guarda relación directa entre el consumo frecuente de hidratos de carbono y la actividad cariogénica."}

\section{ABSTRACT}

In the last decade, the most prevalent diseases in the oral cavity according to WHO and PAHO are dental caries and periodontal disease being these, risk factors for cardiovascular diseases, respiratory, gastrointestinal affecting systems of the human body. WHO he said that 60 to $90 \%$ of school children worldwide suffer from dental caries and $38.7 \%$ suffer from gingivitis. Oral health is important for the physical and mental development of people as it plays an important role in the quality of life. However, tooth decay and periodontal disease appear among the leading causes of oral morbidity worldwide and our country is not free of these problems. Methods: It was a descriptive, prospective and cross-sectional study, whose sample consisted of 248 children of the District of Oropesa I.E. Results: We found a high prevalence of severe caries in $64.3 \%$ followed by moderate caries in $29.5 \%$; due to high consumption of foods high in extrinsic sugars. The frequency of consumption of cariogenic food was high $(63 \%)$ and protective food was low by $55 \%$. The male presented dental caries in $34.4 \%$ and females had higher percentage of gingivitis in $25.4 \%$. Conclusions:

Eating habits are related to the prevalence of caries, due to high consumption of foods high in extrinsic sugars, it is also associated with gingivitis; this is due to lack of knowledge about oral hygiene, also a higher prevalence of severe decay and mild gingivitis was found.

KEYWORDS: Eating Habits, Dental Caries and Gingivitis.

Qs importante considerar que los hábitos alimenticios, higiene y estilos de vida saludable se asimilan e integran a la personalidad durante los primeros años vida, consolidándose hasta perdurar incluso en la edad adulta; de ahí la importancia de brindar una orientación adecuada y oportuna a docentes y padres responsables de la atención y cuidado de los niños, en cuanto a qué y cómo hacer para promover la enseñanza de hábitos saludables y valorarlos como herramientas que ayudan a proteger la salud bucal.(1)

La Organización Mundial de la Salud (OMS) en 2014, reportó que a nivel mundial la prevalencia de caries en niños de 6 a 12 años fue de 60-90\%. El tártaro dental afectó a $23.3 \%$, y la gingivitis afectó al 38.7\% (2). Las enfermedades de mayor prevalencia en la cavidad oral son la caries y gingivitis como factores de riesgo en el desarrollo de patologías que afectan a otros sistemas del cuerpo humano. El Perú es uno de los países de Sudamérica con mayor índice de enfermedades orales, la prevalencia de caries en niños es $80 \%$ y de gingivitis es $70 \%$. (3)

\section{Diversos factores predisponen al} individuo a adquirir caries, como: la calidad de la dieta, la misma que guarda relación directa entre el consumo frecuente de hidratos de carbono y la actividad cariogénica. Hemos observado que los niños comprendidos entre 3 a 14 años, presentan una alta prevalencia de caries dentinaria caracterizada por la destrucción rápida y severa trayendo graves consecuencias a la salud oral y placa bacteriana, placa dental las mismas que son consideradas factores etiológicos de la enfermedad gingival, estos 
problemas conllevan dolor y pérdidas de piezas dentales, que afecta a la apariencia, la calidad de vida, la ingesta de alimentos, y, consecuentemente, al crecimiento y al desarrollo de los niños.

El presente estudio se elaboró debido a que en nuestra ciudad no se encontraron datos relacionados a hábitos alimenticios con la caries y gingivitis.

\section{Materiales y métodos}

Se realizó un estudio descriptivo, prospectivo y transversal, cuya muestra estuvo constituida por 248 niños de sexo masculino y femenino entre 6 y 12 años de edad, que se encontraban debidamente matriculados en las I.E. nacionales y particulares del distrito de Oropesa durante el año 2014. Para el análisis de los resultados se emplearon procedimientos estadísticos estándar y el programa estadístico SPSS 21.

\section{Resultados}

Se encontró una alta prevalencia la caries grave en un $64.3 \%$ seguido de la caries moderada en un $29.5 \%$; debido al elevado consumo de alimentos ricos en azucares extrínsecos.

Tabla 1. Prevalencia de la Caries Dental en Niños de 06-12 años, en las Instituciones Educativas de Oropesa.

\begin{tabular}{lll}
\hline Caries & Frecuencia & Porcentaje \\
\hline Leve & 14 & 6.3 \\
\hline Moderada & 66 & 29.5 \\
\hline Grave & 144 & 64.3 \\
\hline Total & 224 & 100.0
\end{tabular}

Fuente: Ficha de recolección de datos.

Tabla 2. Prevalencia de la Gingivitis en Niños de 06-12 años, en las Instituciones Educativas de Oropesa.

\begin{tabular}{lll}
\hline Gingivitis & Frecuencia & Porcentaje \\
\hline Leve & 68 & 30.4 \\
\hline Moderada & 110 & 49.1 \\
\hline Grave & 46 & 20.5 \\
\hline Total & 224 & 100.0
\end{tabular}

Fuente: Ficha de recolección de datos.
Tabla 3. Frecuencia de Consumo de Alimentos Cariogénicos y Protectores en Niños de 06-12 Años, en las Instituciones Educativas de Oropesa.

\begin{tabular}{lll}
\hline $\begin{array}{l}\text { Hábitos } \\
\text { Alimenticios }\end{array}$ & $\begin{array}{l}\text { Frecuencia de } \\
\text { Consumo de } \\
\text { Alimentos } \\
\text { Cariogénicos }\end{array}$ & $\begin{array}{l}\text { Frecuencia de } \\
\text { Consumo de } \\
\text { Alimentos Protectores }\end{array}$ \\
\hline Bajo & 11.2 & 54.9 \\
\hline Moderado & 25.9 & 35.7 \\
Alto & 2.9 & 9.4 \\
\hline
\end{tabular}

Fuente: Ficha de recolección de datos.

Tabla 4. Distribución Numérica y Porcentual de la Relación entre hábitos Alimenticios y la Caries Dental en Niños de 06-12 Años, en las Instituciones Educativas de Oropesa.

\begin{tabular}{|c|c|c|c|c|c|}
\hline \multirow{2}{*}{$\begin{array}{l}\text { Hábitos } \\
\text { Alimenticios }\end{array}$} & & \multicolumn{3}{|c|}{ Caries } & \multirow[t]{2}{*}{ Total } \\
\hline & & Leve & Moderada & $\begin{array}{l}\text { Grav } \\
\text { e }\end{array}$ & \\
\hline \multirow{8}{*}{$\begin{array}{l}\text { Frecuencia } \\
\text { de Consumo } \\
\text { de Alimentos } \\
\text { Cariogénicos }\end{array}$} & Bajo & 11 & 12 & 2 & 25 \\
\hline & & $5 \%$ & $5 \%$ & $1 \%$ & $11 \%$ \\
\hline & Moderado & 3 & 37 & 18 & 58 \\
\hline & & $1 \%$ & $17 \%$ & $8 \%$ & $26 \%$ \\
\hline & Alto & 0 & 17 & 124 & 141 \\
\hline & & $0 \%$ & $8 \%$ & $55 \%$ & $63 \%$ \\
\hline & Total & 14 & 66 & 144 & 224 \\
\hline & & $6 \%$ & $29 \%$ & $64 \%$ & $100 \%$ \\
\hline \multirow{8}{*}{$\begin{array}{l}\text { Frecuencia } \\
\text { de Consumo } \\
\text { de Alimentos } \\
\text { Protectores }\end{array}$} & Bajo & 1 & 17 & 105 & 123 \\
\hline & & $0 \%$ & $8 \%$ & $47 \%$ & $55 \%$ \\
\hline & Moderado & 0 & 42 & 38 & 80 \\
\hline & & $0 \%$ & $19 \%$ & $17 \%$ & $36 \%$ \\
\hline & Alto & 13 & 7 & 1 & 21 \\
\hline & & $6 \%$ & $3 \%$ & $0 \%$ & $9 \%$ \\
\hline & Total & 14 & 66 & 144 & 224 \\
\hline & & $6 \%$ & $29 \%$ & $64 \%$ & $100 \%$ \\
\hline
\end{tabular}

Fuente: Ficha de recolección de datos.

El consumo de alimentos cariogénicos fue alto en un $63 \%$ y la frecuencia de consumo de alimentos protectores fue baja en un $55 \%$, en ambos casos prevaleció la caries grave en un $64 \%$ lo cual nos indica que a malos hábitos alimenticios se presentó un alto porcentaje de caries grave. 
Tabla 5. Distribución Numérica y Porcentual de la Relación entre Hábitos Alimenticios y la Gingivitis en Niños de 06-12 Años, en las Instituciones Educativas de Oropesa

\begin{tabular}{|c|c|c|c|c|c|}
\hline \multirow{2}{*}{$\begin{array}{l}\text { Hábitos } \\
\text { Alimenticios }\end{array}$} & & \multicolumn{3}{|c|}{ Gingivitis } & \multirow[t]{2}{*}{ Total } \\
\hline & & Sana & Leve & Moderada & \\
\hline \multirow{8}{*}{$\begin{array}{l}\text { Frecuencia d } \\
\text { Consumo de } \\
\text { Alimentos } \\
\text { Protectores }\end{array}$} & \multirow[t]{2}{*}{ Bajo } & 44 & 42 & 37 & 123 \\
\hline & & $20 \%$ & $19 \%$ & $17 \%$ & $55 \%$ \\
\hline & \multirow{2}{*}{ Moderado } & 23 & 48 & 9 & 80 \\
\hline & & $10 \%$ & $21 \%$ & $4 \%$ & $36 \%$ \\
\hline & \multirow[t]{2}{*}{ Alto } & 1 & 20 & 0 & 21 \\
\hline & & $0 \%$ & $9 \%$ & $0 \%$ & $9 \%$ \\
\hline & \multirow[t]{2}{*}{ Total } & 68 & 110 & 46 & 224 \\
\hline & & $30 \%$ & $49 \%$ & $21 \%$ & $100 \%$ \\
\hline & Bajo & $\underline{2}$ & 23 & 0 & 25 \\
\hline \multirow{7}{*}{$\begin{array}{l}\text { Frecuencia de } \\
\text { Consumo de } \\
\text { Alimentos } \\
\text { Cariogénicos }\end{array}$} & & $1 \%$ & $10 \%$ & $0 \%$ & $11 \%$ \\
\hline & \multirow{2}{*}{ Moderado } & 10 & 44 & 4 & 58 \\
\hline & & $4 \%$ & $20 \%$ & $2 \%$ & $26 \%$ \\
\hline & \multirow[t]{2}{*}{ Alto } & 56 & 43 & 42 & 141 \\
\hline & & $25 \%$ & $19 \%$ & $19 \%$ & $63 \%$ \\
\hline & \multirow[t]{2}{*}{ Total } & 68 & 110 & 46 & 224 \\
\hline & & $30 \%$ & $49 \%$ & $21 \%$ & $100 \%$ \\
\hline
\end{tabular}

Fuente: Ficha de recolección de datos.

El consumo de alimentos cariogénicos, fue alto en un $63 \%$ y la frecuencia de consumo de alimentos protectores fue bajo en un $55 \%$, en ambos casos se presentó una alta prevalencia de gingivitis leve que represento un $49 \%$; estos resultados nos indican que a malos hábitos alimenticios se presentó un alto porcentaje de la gingivitis leve.

Tabla 6. Distribución Numérica y Porcentual de la Relación entre la Caries Dental y el Sexo en Niños de 06-12 Años, en las Instituciones Educativas de Oropesa

\begin{tabular}{llll}
\hline Caries & \multicolumn{2}{l}{ Sexo } & \multirow{2}{*}{ Total } \\
\cline { 2 - 3 } & Masculıno & Femenino & \\
\hline Leve & 7 & 7 & 14 \\
\cline { 2 - 4 } & $3.1 \%$ & $3.1 \%$ & $6.3 \%$ \\
\hline Moderada & 32 & 34 & 66 \\
\cline { 2 - 4 } & $14.3 \%$ & $15.2 \%$ & $29.5 \%$ \\
\hline Grave & 77 & 67 & 144 \\
\hline Total & $34.4 \%$ & $29.9 \%$ & $64.3 \%$ \\
\hline
\end{tabular}

Fuente: Ficha de recolección de datos.
Tabla 7. Distribución Numérica y Porcentual de la Relación entre la Gingivitis y el Sexo en Niños De 06-12 Años, en las I.E de Oropesa

\begin{tabular}{llll}
\hline \multirow{2}{*}{ Gingivitis } & \multicolumn{2}{l}{ Sexo } & \multirow{2}{*}{ Total } \\
\cline { 2 - 3 } & Masculino & Femenino & \\
\hline \multirow{2}{*}{ Sana } & 35 & 33 & 68 \\
\cline { 2 - 4 } & $15.6 \%$ & $14.7 \%$ & $30.4 \%$ \\
\hline Leve & 53 & 57 & 110 \\
\cline { 2 - 4 } & $23.7 \%$ & $25.4 \%$ & $49.1 \%$ \\
\hline Moderada & 28 & 18 & 46 \\
\hline & $12.5 \%$ & $8.0 \%$ & $20.5 \%$ \\
\hline \multirow{2}{*}{ Total } & $51.8 \%$ & $48.2 \%$ & $100.0 \%$ \\
\hline
\end{tabular}

Fuente: Ficha de recolección de datos.

Tabla 8. Distribucion Numérica y Porcentual de la Relación entre Caries Dental y la Institución Educativa en Niños de 06-12 Años, en las I.E de Oropesa.

\begin{tabular}{lllll}
\hline \multirow{2}{*}{$\begin{array}{l}\text { Institución } \\
\text { Educativa }\end{array}$} & \multicolumn{2}{l}{ Caries } & & \multicolumn{2}{l}{ Total } \\
\cline { 2 - 4 } & Leve & Moderada & Grave & \\
\hline Particular & 5 & 29 & 25 & 59 \\
\cline { 2 - 5 } & $2 \%$ & $13 \%$ & $11 \%$ & $26 \%$ \\
\hline Nacional & 9 & 37 & 119 & 165 \\
\cline { 2 - 5 } & $4 \%$ & $17 \%$ & $53 \%$ & $74 \%$ \\
\hline Total & 14 & 66 & 144 & 224 \\
\hline
\end{tabular}

Fuente: Ficha de recolección de datos.

Tabla 9. Distribución Numérica y Porcentual de la Relación entre la Gingivitis y la Institución Educativa en Niños de 06-12 Años, en las I.E de Oropesa

\begin{tabular}{lllll}
\hline Institución & \multicolumn{2}{l}{ Gingivitis } & \multicolumn{2}{c}{ Total } \\
\cline { 2 - 5 } Educativa & Sana & Leve & \multicolumn{2}{c}{ Moderada } \\
\hline Particular & 15 & 37 & 7 & 59 \\
\cline { 2 - 5 } & $6 \%$ & $17 \%$ & $3 \%$ & $26 \%$ \\
\hline Nacional & 53 & 73 & 39 & 165 \\
\cline { 2 - 5 } & $24 \%$ & $33 \%$ & $17 \%$ & $74 \%$ \\
\hline \multirow{2}{*}{ Total } & 68 & 110 & 46 & 224 \\
\cline { 3 - 5 } & $30 \%$ & $49 \%$ & $21 \%$ & $100 \%$ \\
\hline
\end{tabular}

Fuente: Ficha de recolección de datos.

\section{Discusión}

El presente estudio tuvo como propósito determinar la relación entre los hábitos alimenticios con caries y la 
gingivitis en niños de las Instituciones

Educativas del distrito de Oropesa. Este estudio se realizó en 224 niños, obteniendo resultados estadísticamente significativos.

En el presente estudio se encontró que el $100 \%$ de los niños de 6 a 12 años tuvieron una alta experiencia de caries, siendo el más prevalente el índice grave $(\mathrm{CPOD}=7$ en un $64 \%)$, siendo el grupo más afectado el sexo masculino el que presentó caries grave fue de $34.4 \%$; resultados similares con los estudios realizados por: velarde $(56.3 \%)$, Ponce $(81.8 \%)$, Flores M. y Montenegro B. $(80 \%)$, Ramos $(88.7 \%)$ y difiere del estudio realizado por Lara $(11.60 \%)$ esto puede deberse a que su estudio fue realizado en una población diferente, con un rango etario de 6 a 36 meses y al hecho de que el Estado de Chihuahua es una zona con alto contenido de flúor en el agua, lo que contribuye a la prevención de la caries. En consecuencia podemos deducir que todos los programas de salud bucal están enfocados principalmente al segundo nivel de prevención, y no así al primer nivel siendo este el más importante para prevenir la caries y la gingivitis.

También se encontró una alta prevalencia de gingivitis leve (49\%); siendo el grupo más afectado el sexo masculino $(36.2 \%)$, dicho resultado difiere al estudio realizado por: Velarde $(4,20 \%)$ esto puede ser debido a que la población tuvo una mayor frecuencia del cepillado dental.

En cuanto a la frecuencia del consumo de alimentos cariogénicos se observó una frecuencia alta $(63 \%)$ y una frecuencia baja del consumo de alimentos protectores (55\%); existiendo una relación entre estos y la prevalencia de caries, esto debido a que los niños están más expuestos a alimentos que contienen azúcares extrínsecos. La caries dental, es considerada también como una enfermedad dieto-bacteriana, ya que en la ausencia de placa o de carbohidratos y la dieta no se desarrolla. Es por ello que, los carbohidratos son uno de los factores etiológicos más importantes de la caries dental. A sí mismo, Barrancos Money (15) señala que la dieta contribuye al desarrollo de la caries. Cuyos resultados son similares con los estudios realizados por: Ponce y Ramos. Difieren a los estudios realizados por: Velarde; el cual obtuvo que no hubo relación entre los hábitos alimenticios con la caries dental.

"El nivel socioeconómico bajo está estrechamente relacionado con una deficiencia de higiene oral, malos hábitos alimenticios y falta de conocimiento sobre el cuidado de la salud bucal.'

Así mismo, se encontró que existe una relación entre ambas Instituciones Educativas con la caries dental, gingivitis y la higiene oral siendo estos más prevalentes en la Institución Educativa Nacional; la caries grave (53\%), gingivitis leve $(33 \%)$ y una mala higiene oral $(54.9 \%)$; demostrándose que el nivel socioeconómico bajo está estrechamente relacionado con una deficiencia de higiene oral, malos hábitos alimenticios y falta de conocimiento sobre el cuidado de la salud bucal. 


\section{Conclusiones}

Los hábitos alimenticios están relacionados con la prevalencia de caries, debido al elevado consumo de alimentos ricos en azucares extrínsecos, también está relacionado con la gingivitis; esto se debe a la falta de conocimientos sobre la higiene oral.

La población escolar estudiada presentó una mayor prevalencia de la caries grave y de una gingivitis leve.

La frecuencia de consumo de alimentos cariogénicos fue alto debido a que la población presentaron un elevado consumo de hidratos de carbono propios de la región, y la frecuencia de consumo de alimentos protectores fue baja.

La prevalencia de caries y gingivitis no está relacionado con el sexo, tanto el masculino y el femenino presentaron un alto porcentaje de caries grave y gingivitis leve. No se evidencio una relación entre la higiene oral y el sexo porque en ambos sexos presentaron una higiene oral deficiente.

Se encontró una relación entre la caries, gingivitis y la higiene oral con la Institución Educativa siendo estas más prevalentes en la Institución Educativa Nacional lo cual evidencia una relación directa con el nivel socioeconómico bajo.

\section{REFERENCIAS}

(1) Gimenez Mondragon N. Nutrición y Alimentación su Relación con la Salud Bucal en pacientes adultos mayores que acuden al Hospital y al consultorio privado en la ciudad Calchaquí; México. Universidad Abierta Interamericana. 2011, pág. 15-25.

(2) Lara Gonzales A. Caries y su Relación con Hábitos Alimenticios y de Higiene en niños de 6 a 36 meses de edad, México: Universidad Autónoma de Chihuahua 2012, pág. 23-37.

(3) Maita L. Promoción de Salud Bucal en Alumnos y Trabajadores Administrativos y de Servicio de la Facultad de Medicina de la Universidad Nacional Mayor de San Marcos. Odontol. Sanmarquina 2006, pág. 32-47.

(4) Velarde Grados I. Estado de Salud Bucodental de Escolares de Primero de primaria del Distrito la Ciudad Lineal de Madrid y su Relación con Hábitos de Alimentación y Parámetros de Salivación. Madrid: Universidad Complutense 2013, pág. 31-49.

(5) Ponce Caceres C. Prevalencia de Caries Dental y su Relación con los Hábitos Alimenticios y de Higiene Bucal en Infantes de 06 a 36 meses de edad en el Programa CRED, distritos de Hunter y Socabaya, Universidad Católica de Santa María. 2010, pág. 24-39.

(6) Flores Moreno M, Montenegro Gutiérrez B. Relación entre la Frecuencia Diaria de Consumo de Azúcares Extrínsecos y la Prevalencia de Caries Dental Atendidos en la Clínica Estomatológica Central de la Universidad Peruana Cayetano Heredia 2003, pág. 29-38.

(7) Ramos Coello K. Influencia del Contenido de la Lonchera Pre-Escolar como Factor Predisponente de Caries Dental en Niños de 3 a 5 años de edad de la Institución Educativa PNP STA. Rosita de Lima y del Jardín Funny Garde, Universidad Católica de Santa María.2009, pag. 21-39.

(8) Higashida B. Odontología Preventiva 1era ed. McGraw-Hill interamericana: 2000, pág. 103-117.

(9) Barrancos M. Operatoria Dental. 4ta edición. Argentina: Editorial Médica Panamericana. 2006, pág. 251-267.

(10) Gutierrez I V. Promoción de la Salud Bucodental. Prevlnfad/PAPPS 2011, pág. 33-45.

(11) Santillán Jimenez M. Nivel de Conocimiento y Conducta Sobre la Salud Bucal en internos de ciencias de salud, Universidad Nacional Mayor de San Marcos. Odontol. Sanmarquina 2009, pág. 15-19. 